\title{
A ternary decision diagram method to calculate the component contributions to the failure of systems undergoing phased missions
}

\author{
J D Andrews \\ Department of Aeronautical and Automotive Engineering, Loughborough University, \\ Loughborough, Leicestershire LE11 3TU, UK. email: J.D.Andrews@lboro.ac.uk
}

The manuscript was received on 15 May 2007 and was accepted after revision for publication on 12 February 2008.

DOI: $10.1243 / 1748006 X J R R 92$

\begin{abstract}
The way that many systems are utilized can be expressed in terms of missions which are split into a sequence of contiguous phases. Mission success is only achieved if each of the phases is successful, and each phase is required to achieve a different objective and use different elements of the system. The reliability analysis of a phased mission system will produce the probability of failure during each of the phases, together with the overall mission failure likelihood. In the event that the system performance does not meet with the acceptance requirement, weaknesses in the design are identified and improvements made to rectify the deficiencies. In conventional system assessments, importance measures can be predicted which provide a numerical indicator of the significance of the role that each component plays in the system failure. Through the development of appropriate importance measures, this paper provides ways of identifying the contribution made by each component failure to each phase failure and the overall mission failure. In addition, a means is given to update the system performance prediction as phases of the mission are successfully completed. The causes of phase failure are expressed as fault trees. The binary decision diagram (BDD) concept is extended to produce ternary decision diagrams (TDDs) to facilitate fast calculation of the importance measures.
\end{abstract}

Keywords: phased mission analysis, importance measures, binary decision diagrams, ternary decision diagrams, fault tree analysis

\section{INTRODUCTION}

A phased mission is used to describe the situation where the system functional requirements change throughout the period of operation. The periods of operation between the transition points, where the system functional requirements change, are referred to as phases, all of which need to be completed successfully for the mission success. Many systems can be seen to operate in this way; typical examples are aircraft, satellites, and spacecraft.

The unreliability assessment of phased mission systems produces the likelihood of failure during each of the individual phases and also the overall mission failure probability. For some systems it may be possible for maintenance to be performed during the mission to rectify faults that have occurred. The categorization of a mission to be non-repairable or repairable influences the reliability modelling techniques that can be used. Fault tree approaches are appropriate for non-repairable phased missions [1-3], and Markov methods when some degree of repair is possible [4-6]. This paper focuses on nonrepairable missions.

Recent work has extended the basic mission unreliability modelling methods to indicate the contribution that individual components can make to the mission failure $[\mathbf{7}, \mathbf{8}]$. The development of these importance measures has extended the concepts of the classical Birnbaum measure of importance and the criticality measure of importance [9, 10]. The contributions made to each phase failure are calculated with account taken of the fact that failure in a phase can only occur providing all previous phases have successfully completed. The phase failure contributions are then combined to give an indication of the contribution that each component failure makes when considering the entire mission. 
As the mission progresses and each phase is successfully completed, the predictions for the phase and mission failure probability can be updated. The method to perform the revised predictions is given.

For a non-repairable mission, the causes of phase failure can be expressed as a fault tree. It has been shown that binary decision diagram (BDD) methods [11-13] can offer an efficient and accurate solution to these problems even in a phased mission context [3]. However, to calculate the importance contributions, these need to be extended to the ternary decision diagram (TDD).

\section{PHASED MISSION DEFINITION}

In the modelling presented in this paper, the following assumptions are made for the phased mission.

1. A mission is defined in terms of phases carried out consecutively.

2. Each phase accomplishes a specified task. It has different functional requirements and therefore the failure criteria are different for each phase.

3. For mission success, each phase must be completed successfully.

4. The time duration for each phase is known.

5. The mission is non-repairable and component failures will exist for the remainder of the mission once they occur.

6. All components are in the working state at the start of the mission.

\section{PHASED MISSION UNRELIABILITY QUANTIFICATION}

The phased mission is represented as a series of fault trees, each one expressing the conditions that will lead to the failure of a specific phase. The duration of each phase is also provided in terms of the times, following the mission initiation, at which each phase is entered. A method to calculate the failure likelihood of such a phased mission, the phased mission unreliability $Q_{\text {sys }}$, is presented in reference [3]. It provides both qualitative and quantitative information regarding phase and mission failure. The method presented in this paper breaks down the phase failure modes to identify where the significant contributions occur.

Component failures are considered as separate, dependent events in each phase. The notation used is as follows: $A_{i}$ represents the failure of component $A$ during phase $i$, and $A_{i, j}$ represents the component failing at some point between the start of phase $i$ and the end of phase $j$. Therefore, the event that the component exists in the failed state at the end of phase $i$ is

$$
A_{1, i}=A_{1}+A_{2}+\cdots+A_{i}
$$

In all logic equations, ' + ' is used to represent $\mathrm{OR}$ and $\because$ ' is used to represent AND.

The first stage of the method is to establish the phase failure modes. These are the prime implicants minimal combinations of the component states (working or failed) that will result in a particular phase failure and account for the successful completion of all previous phases. Considering each phase in turn, the method constructs the phase failure fault tree as shown in Fig. 1. Boolean reduction of the fault trees constructed in this way determines the phase failure modes. In performing the reduction for a phased mission fault tree, it is possible to take advantage of the non-repairable nature of the component failures. A special phase algebra has been developed that uses the fact that, once failed, a component remains that way for the rest of the mission. The algebra uses the following rules

$$
\begin{aligned}
& A_{i} \cdot A_{j}=0 \\
& A_{i} \cdot A_{i}=A_{i} \\
& A_{i} \cdot A_{i, j}=A_{i} \\
& A_{i} \cdot \bar{A}_{i}=0 \\
& \bar{A}_{i} \cdot A_{i, j}=A_{i+1, j} \\
& A_{i} \cdot \bar{A}_{j}=0 \quad \text { if } i<j
\end{aligned}
$$

In a phased mission there are two ways that a phase can experience a failure.

1. The failure can occur during the phase as a result of a component failure that occurs during the phase, the occurrence of which then fulfils the conditions for phase and therefore mission failure.

2. Alternatively, the system can be in a state that already satisfies the conditions for phase failure before the phase is entered. Phase failure will then result as soon as the transition into the phase takes place. In this latter case the component failure events in the phase failure mode have all occurred in a previous phase but have not satisfied a previous phase failure condition.

The phase failure modes can be split into the causes of these two categories: in-phase failure and phase transition failure.

The likelihood of the component failure events that appear in the prime implicants obtained for each phase fault tree can be determined by integrating their failure time density function over the appropriate time period

$$
q_{A_{i, j}}=\int_{t_{i-1}}^{t_{j}} f_{A}(t) \mathrm{d} t
$$

From the component failure probabilities, together with the prime implicant sets, the inclusion-exclusion expansion (equation (4)) can be used to determine 


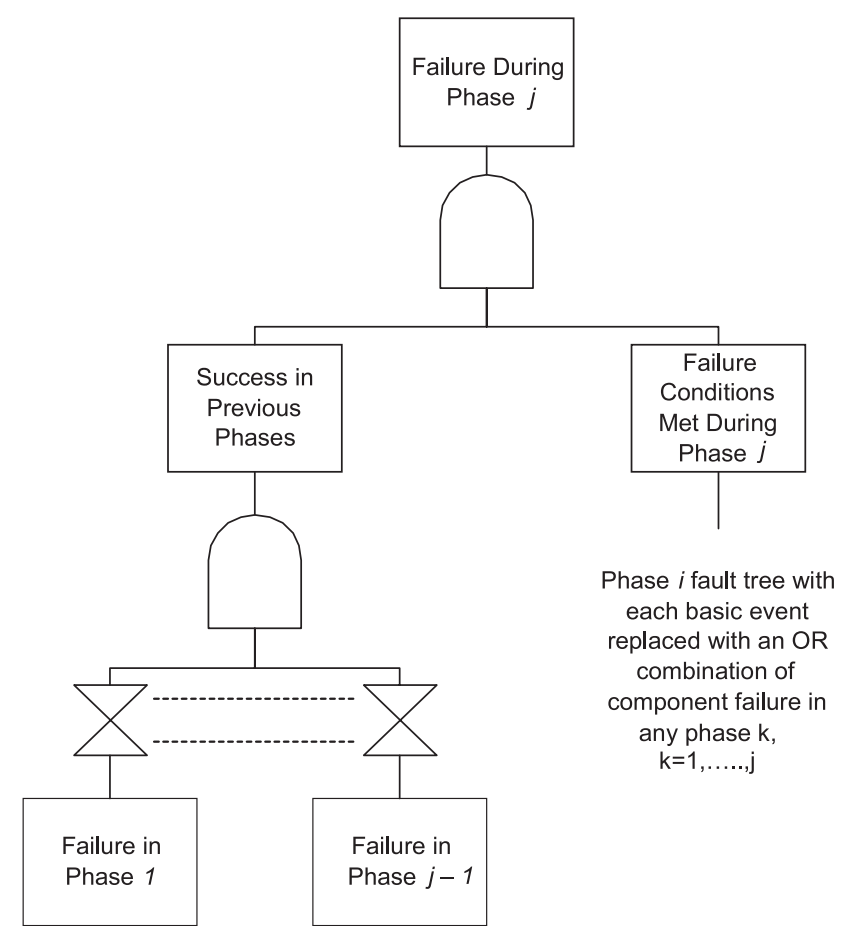

Fig. 1 Fault tree for mission failure during phase $i$

the phase $j$ unreliabilities (conditional on all previous phase success) $Q_{j}^{\mathrm{P}}$ and $Q_{j}^{\mathrm{T}}$, the in-phase and phase transition failure probabilities respectively

$$
\begin{aligned}
Q_{j}^{\mathrm{P} / \mathrm{T}}= & \sum_{i=1}^{N_{\mathrm{p}}} P\left(C_{i}\right)-\sum_{i=1}^{N_{\mathrm{p}}} \sum_{k=1}^{i-1} P\left(C_{i} \cap C_{k}\right) \ldots \\
& +(-1)^{N_{\mathrm{p}}-1} P\left(C_{1} \cap C_{2} \cap \ldots \cap C_{N_{\mathrm{p}}}\right)
\end{aligned}
$$

where $C_{i}$ is prime implicant $i$.

The phase unreliability is then obtained from

$$
Q_{j}=Q_{j}^{\mathrm{T}}+Q_{j}^{\mathrm{P}}
$$

As will be discussed in later sections, to evaluate the component importance contributions, a particular formulation of equation (4) $[\mathbf{1 4}, \mathbf{1 5}]$ offers some advantages. While the form of the equation is unimportant for phase failure likelihood quantification, the form that will be used later in the importance calculations will be described, and all expressions used in the examples will be formed this way. Prime implicant terms, $C_{i}$, will be of a form that can contain components failing and functioning through particular phases. This form will also describe the combinations of the prime implicants whose likelihood is to be determined in equation (4). The terms of the inclusion-exclusion expansion are formed using two independent variables for the likelihood that any component $i$ works, $p_{i}$, and that it fails, $q_{i}$. The relationship $p_{i}+q_{i}=1$ is not used to express the whole equation in terms of either one of these variables.
For example, if it is required to evaluate the probability of the combination of component failure events $A_{3} \cdot \overline{B_{3}} \cdot C_{2}$, then the form for its probability would be $q_{A_{3}} p_{B_{3}} q_{C_{2}}$. Clearly, the Boolean reduction carried out will prevent situations where the same component exists in both its working and failed state.

Summing the phase unreliabilities yields the mission unreliability

$$
Q_{\text {miss }}=\sum_{\substack{\text { all } \\ \text { phases } \\ j}} Q_{j}
$$

The phase and mission quantification is illustrated using a simple example system provided in the next section. This example will be used throughout the paper and will demonstrate the computation of the component importance measures developed in the later sections.

\section{EXAMPLE SYSTEM ANALYSIS}

An example four-phased mission system is illustrated in Fig. 2. The failure conditions for each of the four phases, in terms of the four components $A, B, C$, and $D$ on which this mission depends, are represented by fault trees. The time durations in each phase $j$ are $\left(t_{j-1}, t_{j}\right)$.

In the system analysis that follows, $\overline{A_{j}}$ represents the functioning of component $A$ throughout phase $j$, 


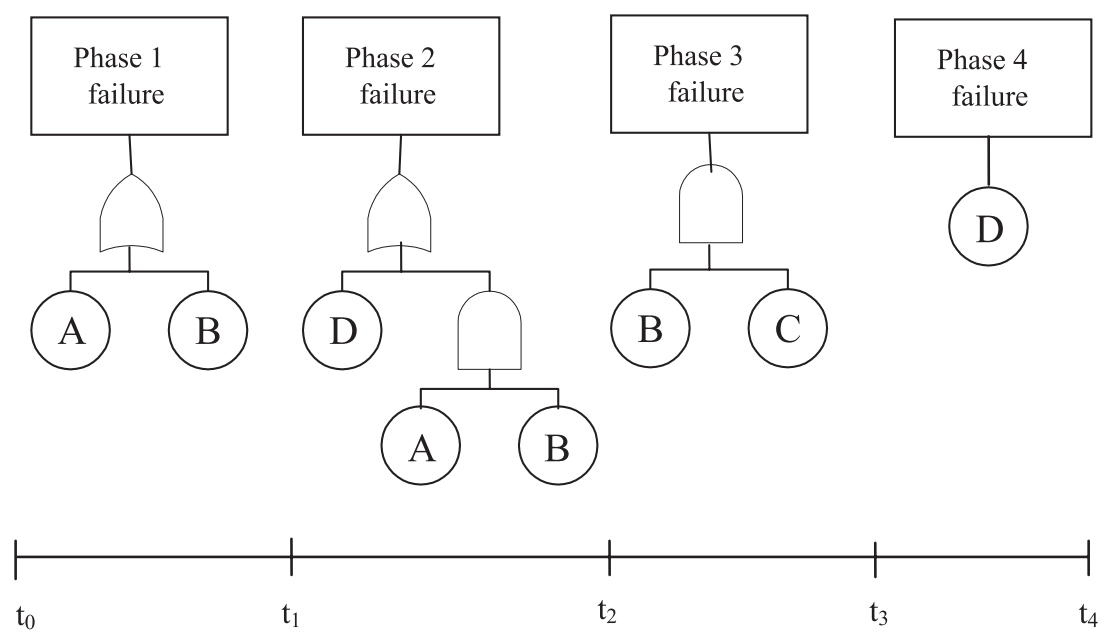

Fig. 2 Example system phased mission fault trees

and, extending this across several phases, $\overline{A_{i, j}}$ represents the functioning of component $A$ throughout phases $i$ to $j$ inclusive.

Prior to obtaining the phase failure probabilities $Q_{j}$, the in-phase failure probability $Q_{j}^{\mathrm{P}}$ and the phase transition failure probability $Q_{j}^{\mathrm{T}}$ must be quantified. This requires the phase failure modes for each of these events.

Reduction of the fault tree shown in Fig. 1 will yield all causes of phase failure, which will include both in-phase and phase transition causes combined in the phase failure modes. The approach taken in this paper determines the in-phase and phase transition failure modes separately. First, the combined failure modes are produced by applying Boolean reduction to the fault tree of the form shown in Fig. 1. Then the phase transition failure modes are developed. Then removing the phase transition causes from the combined phase causes will provide the in-phase failure modes.

\subsection{Combined failure modes}

\subsubsection{Phase 1}

There are no previous phases and so this phase can be treated as a normal non-phased mission system. The logic expression for the causes of failure in phase 1 , $P h_{1}$, and the likelihood of this event, $Q_{1}$, are given by

$$
\begin{aligned}
& P h_{1}=A_{1}+B_{1} \\
& Q_{1}=q_{A_{1}}+q_{B_{1}}-q_{A_{1}} q_{B_{1}}
\end{aligned}
$$

\subsubsection{Phase 2}

In constructing the fault tree for mission failure during phase 2, it will combine the causes of success in phase $1,\left(\overline{\mathrm{A}_{1}+B_{1}}=\overline{A_{1}} \cdot \overline{B_{1}}\right)$, and the failure conditions for phase 2 being met in phase 2, $\left(A_{1,2} \cdot B_{1,2}+D_{1,2}\right)$. The failure logic expression and failure likelihood during phase 2 are

$$
\begin{aligned}
P h_{2} & =\overline{A_{1}} \cdot \overline{B_{1}} \cdot\left[A_{1,2} \cdot B_{1,2}+D_{1,2}\right] \\
& =A_{2} \cdot B_{2}+\overline{A_{1}} \cdot \overline{B_{1}} \cdot D_{1,2} \\
Q_{2} & =q_{A_{2}} q_{B_{2}}+p_{A_{1}} p_{B_{1}} q_{D_{1,2}}-q_{A_{2}} q_{B_{2}} q_{D_{1,2}}
\end{aligned}
$$

\subsubsection{Phase 3}

The logic expression for phase 3 is constructed using the causes of successful completion of phase 1 and successful completion of phase 2 and the failure conditions of phase 3 being met in phase 3

$$
\begin{aligned}
P h_{3} & =\overline{A_{1}} \cdot \overline{B_{1}} \cdot\left(\overline{A_{1,2} \cdot B_{1,2}+D_{1,2}}\right) \cdot\left[B_{1,3} \cdot C_{1,3}\right] \\
& =\overline{A_{1,2}} \cdot B_{2,3} \cdot C_{1,3} \cdot \overline{D_{1,2}}+\overline{A_{1}} B_{3} C_{1,3} \overline{D_{1,2}} \\
& =\overline{A_{1,2}} \cdot B_{3} \cdot C_{1,3} \cdot \overline{D_{1,2}}+\overline{A_{1,2}} \cdot B_{2} \cdot C_{1,3} \cdot \overline{D_{1,2}}+\overline{A_{1}} B_{3} C_{1,3} \overline{D_{1,2}} \\
& =\overline{A_{1,2}} \cdot B_{2} \cdot C_{1,3} \cdot \overline{D_{1,2}}+\overline{A_{1}} B_{3} C_{1,3} \overline{D_{1,2}} \\
Q_{3} & =p_{A_{1,2}} q_{B_{2}} q_{C_{1,3}} p_{D_{1,2}}+p_{A_{1}} q_{B_{3}} q_{C_{1,3}} p_{D_{1,2}}
\end{aligned}
$$

The logic expressions for the phase failure causes are most efficiently dealt with if they are reduced to their minimal form. This involves removing from the expression any replicated or non-minimal terms of the base failure conditions. As an example of this process, consider the expression for $P h_{3}$ in equation (9) (this is the first phase that is not in its minimal form on initial expansion). Expanding out terms from the original expression gives the second line of equation (9). The first term could be expanded out in terms of either $B_{2,3}=B_{2}+B_{3}$ or $C_{1,3}=C_{1}+C_{2}+C_{3}$. Selecting to expand about $B$ gives the third line. Comparing the first and third terms of line 3 shows that the first term can be deleted, as the conditions on $A$ functioning into phase 2 are not necessary. The minimization gives the final logic expression, line 4. 


\subsubsection{Phase 4}

Repeating the procedure for phase 4 gives, for completeness, the results

$$
\begin{aligned}
P h_{4}= & \overline{A_{1,2}} \cdot \overline{B_{1}} \cdot \overline{C_{1,3}} \cdot D_{3,4}+\overline{A_{1}} \cdot \overline{B_{1,3}} \cdot D_{3,4}+\overline{A_{1}} \cdot \overline{B_{1,2}} \cdot \overline{C_{1,3}} \cdot D_{3,4} \\
Q_{4}= & p_{A_{1,2}} p_{B_{1}} p_{C_{1,3}} q_{D_{3,4}}+p_{A_{1}} p_{B_{1,3}} q_{D_{3,4}}+p_{A_{1}} p_{B_{1,2}} p_{C_{1,3}} q_{D_{3,4}} \\
& -p_{A_{1,2}} p_{B_{1,2}} p_{C_{1,3}} q_{D_{3,4}}-p_{A_{1}} p_{B_{1,3}} p_{C_{1,3}} q_{D_{3,4}}
\end{aligned}
$$

\subsection{Phase transition failure modes and probabilities}

The phase transition failure modes can be obtained by modifying the top event in the fault tree structure shown in Fig. 1. Failure on transition to phase $j$ requires success in the previous phases (as in the original structure) AND the failure conditions for phase $j$ are met prior to phase $j$. This latter input branch to the top event fault tree is formed in the same way as before, except component failures are only expanded up to the previous phase, i.e. $A_{1}+A_{2}+\ldots+A_{j-1}$.

The phase transition failure modes $P h_{j}^{\mathrm{T}}$ and probabilities $Q_{j}^{\mathrm{T}}$ for the simple example system shown in Fig. 2 are as follows

Phase 2 transition failure

$$
\begin{aligned}
& P h_{2}^{\mathrm{T}}=\overline{A_{1}} \cdot \overline{B_{1}} \cdot\left(A_{1} \cdot B_{1}+D_{1}\right)=\overline{A_{1}} \cdot \overline{B_{1}} \cdot D_{1} \\
& Q_{2}^{\mathrm{T}}=p_{A_{1}} p_{B_{1}} q_{D_{1}}
\end{aligned}
$$

Phase 3 transition failure

$$
\begin{aligned}
P h_{3}^{\mathrm{T}} & =\overline{A_{1}} \cdot \overline{B_{1}} \cdot\left(\overline{A_{1,2}}+\overline{B_{1,2}}\right) \cdot \overline{D_{1,2}} \cdot\left[B_{1,2} \cdot C_{1,2}\right] \\
& =\overline{A_{1,2}} \cdot B_{2} \cdot C_{1,2} \cdot \overline{D_{1,2}} \\
Q_{3}^{\mathrm{T}} & =p_{A_{1,2}} q_{B_{2}} q_{C_{1,2}} p_{D_{1,2}}
\end{aligned}
$$

Phase 4 transition failure

$$
\begin{aligned}
P h_{4}^{\mathrm{T}}= & \overline{A_{1,2}} \cdot \overline{B_{1}} \cdot \overline{C_{1,3}} \cdot D_{3}+\overline{A_{1}} \cdot \overline{B_{1,3}} \cdot D_{3}+\overline{A_{1}} \cdot \overline{B_{1,2}} \cdot \overline{C_{1,3}} \cdot D_{3} \\
Q_{4}^{\mathrm{T}}= & p_{A_{1,2}} p_{B_{1}} p_{C_{1,3}} q_{D_{3}}+p_{A_{1}} p_{B_{1,3}} q_{D_{3}}+p_{A_{1}} p_{B_{1,2}} p_{C_{1,3}} q_{D_{3}} \\
& -p_{A_{1,2}} p_{B_{1,2}} p_{C_{1,3}} q_{D_{3}}-p_{A_{1}} p_{B_{1,3}} p_{C_{1,3}} q_{D_{3}}
\end{aligned}
$$

\subsection{In-phase failure modes and probabilities}

For each phase, the in-phase failure modes are obtained by removing the phase transition failure modes from the combined failure modes. These are given below, together with the associated probabilities

$$
\begin{aligned}
P h_{2}^{\mathrm{P}} & =\left(A_{2} \cdot B_{2}+\overline{A_{1}} \cdot \overline{B_{1}} \cdot D_{1,2}\right)-\overline{A_{1}} \cdot \overline{B_{1}} \cdot D_{1} \\
& =A_{2} \cdot B_{2}+\overline{A_{1}} \cdot \overline{B_{1}} \cdot D_{2} \\
Q_{2}^{\mathrm{P}} & =q_{A_{2}} q_{B_{2}}+p_{A_{1}} p_{B_{1}} q_{D_{2}}-q_{A_{2}} q_{B_{2}} q_{D_{2}} \\
P h_{3}^{\mathrm{P}} & =\overline{A_{1,2}} \cdot B_{2} \cdot C_{1,3} \cdot \overline{D_{1,2}}+\overline{A_{1}} \cdot B_{3} \cdot C_{1,3} \cdot \overline{D_{1,2}} \\
& \quad-\overline{A_{1,2}} \cdot B_{2} \cdot C_{1,2} \cdot \overline{D_{1,2}} \\
= & \overline{A_{1,2}} \cdot B_{2} \cdot C_{3} \cdot \overline{D_{1,2}}+\overline{A_{1}} \cdot B_{3} \cdot C_{1,3} \cdot \overline{D_{1,2}}
\end{aligned}
$$

$$
Q_{3}^{\mathrm{P}}=p_{A_{1,2}} \cdot q_{B_{2}} \cdot q_{C_{3}} \cdot p_{D_{1,2}}+p_{A_{1}} \cdot q_{B_{3}} \cdot q_{C_{1,3}} \cdot p_{D_{1,2}}
$$

$$
\begin{aligned}
& P h_{4}^{\mathrm{P}}= \\
& {\left[\overline{A_{1,2}} \cdot \overline{B_{1}} \cdot \overline{C_{1,3}} \cdot D_{3,4}+\overline{A_{1}} \cdot \overline{B_{1,3}} \cdot D_{3,4}+\overline{A_{1}} \cdot \overline{B_{1,2}} \cdot \overline{C_{1,3}} \cdot D_{3,4}\right]} \\
& \quad-\left[\overline{A_{1,2}} \cdot \overline{B_{1}} \cdot \overline{C_{1,3}} \cdot D_{3}+\overline{A_{1}} \cdot \overline{B_{1,3}} \cdot D_{3}+\overline{A_{1}} \cdot \overline{B_{1,2}} \cdot \overline{C_{1,3}} \cdot D_{3}\right] \\
& \quad=\overline{A_{1,2}} \cdot \overline{B_{1}} \cdot \overline{C_{1,3}} \cdot D_{4}+\overline{A_{1}} \cdot \overline{B_{1,3}} \cdot D_{4}+\overline{A_{1}} \cdot \overline{B_{1,2}} \cdot \overline{C_{1,3}} \cdot D_{4}
\end{aligned}
$$

$$
\begin{gathered}
Q_{4}^{P}=p_{A_{1,2}} p_{B_{1}} p_{C_{1,3}} q_{D_{4}}+p_{A_{1}} p_{B_{1,3}} q_{D_{4}}+p_{A_{1}} p_{B_{1,2}} p_{C_{1,3}} q_{D_{4}} \\
-p_{A_{1,2}} p_{B_{1,2}} p_{C_{1,3}} q_{D_{4}}-p_{A_{1}} p_{B_{1,3}} p_{C_{1,3}} q_{D_{4}}
\end{gathered}
$$

In equations (14) and (18) the algebra required for the failure mode subtraction is obvious. In equation (16) the process is not as transparent, and each term is first expanded to its fundamental failure modes expressed in terms of single phase variables prior to performing the subtraction.

\section{COMPONENT IMPORTANCE MEASURES}

The criticality measure of importance identifies the contribution that each component makes to the system failure. This concept will be extended to the phased mission context to produce the importance measures for components for both phase and mission failure. In order to calculate the criticality measure, the likelihood of the system being critical for each component needs to calculated (Birbaum's measure of importance). This needs the concept of a critical system state, which for non-phased missions is defined as follows: A critical system state for component $i$ is a state of the remaining components in the system such that the failure of component $i$ will cause the system to make a transition from the working state to the failed state.

The probability that the system is in a critical system state for any component is Birnbaum's measure of importance, $G_{i}$, and can be calculated from

$$
G_{i}=\frac{\partial Q_{\text {sys }}}{\partial q_{i}}
$$

From this, the criticality measure of importance can be determined. It is the probability that the system is in a critical state for component $i$ and that component $i$ has failed. This is normalized by dividing by the system failure probability. This calculates the likelihood that component $i$ has caused the system failure. The criticality measure of importance for component $i$ is given by

$$
I_{i}=\frac{G_{i} q_{i}}{Q_{\mathrm{sys}}}
$$




\section{CRITICAL PHASE STATES}

\subsection{In-phase failure}

For multiphased missions the possible component states in any phase are dependent upon which failures have occurred during all the preceding phases up to and including phase $j$.

A critical phase state for component $i$ in phase $j$ is a state of the remaining components through the previous and current phases such that the system is working on entry to phase $j$, and failure of component $i$ during phase $j$ will cause the phase (and mission) failure.

For this to happen:

(a) all phases up to phase $j$ must have been completed successfully;

(b) component $i$ must be in the working state on entry to phase $j$.

As an example, consider the critical phase states for component $A$ in phase 2 for the simple example system shown in Fig. 2. In phase 2 the system state is determined by the state of components $A, B$, and $D$. In evaluating the critical phase states for $A$, consideration must be given to the states of components $B$ and $D$ through phase 2 and the preceding phase 1 . There are three options for each of the components: they can fail in phase 1 , fail in phase 2 , or work throughout both phases. For the two components, this gives nine states to consider, which are listed in the first column of Table 1. Any combination of states that includes $B$ failed in phase 1 will result in phase 1 failure and does not need to be considered in phase 2 . In phase 2 , if $D$ has failed then phase 2 fails regardless of the state of component $A$, and so these combinations are not critical for $A$. It is only when $D$ is working and $B$ has already failed that it is critical for $A$, which is just the one combination in the last row. The probability of this combination is the criticality for component $A$, in phase 2 , i.e.

$$
G_{A, 2}=q_{B_{2}} p_{D_{1,2}}
$$

where $G_{i, j}$ denotes the criticality function of component $i$ in phase $j$.
As the number of components and the number of phases increases, this tabular approach soon becomes impractical and another derivation of the criticality function is required.

$$
\begin{aligned}
& G_{i, j}=P(\text { system is critical for component } \\
& i \text { in phase } j \text { and the system has } \\
& \text { survived to phase } j \text { and } \\
& \text { component } i \text { is working) } \\
& =P(\text { system has failed in phase } j \\
& \text { with component } i \text { failing in phase } j \\
& \text { and the system surviving to phase } j \text { ) } \\
& \text { - } P \text { (system has failed in phase } j \\
& \text { with component } i \text { working } \\
& \text { throughout phase } j \text { and the } \\
& \text { system surviving to phase } j \text { ) } \\
& =Q_{j}\left(\boldsymbol{q}, q_{i_{j}}=1\right)-Q_{j}\left(\boldsymbol{q}, q_{i_{j}}=0\right) \\
& =\frac{\partial Q_{j}}{\partial q_{i_{j}}}
\end{aligned}
$$

The last step above is true, as $Q_{j}$ is a linear function of $q_{i_{j}}$. Note that the expression for $Q_{j}$ derived from the combined phase failure modes is used. This is needed in order to account correctly for the failure not occurring on entry to the phase. For example

\begin{tabular}{|c|c|c|c|}
\hline $\begin{array}{l}\text { Other component } \\
\text { states }\end{array}$ & $\begin{array}{l}\text { Fails in } \\
\text { phase } 1 ?\end{array}$ & $\begin{array}{l}\text { Critical for } \\
\text { component } \\
A \text { in phase } 2 ?\end{array}$ & Probability \\
\hline$\overline{B_{1,2}} \cdot D_{1}$ & No & No & - \\
\hline$\overline{B_{1,2}} \cdot D_{2}$ & No & No & - \\
\hline$\overline{B_{1,2}} \cdot \overline{D_{1,2}}$ & No & No & - \\
\hline$B_{1} \cdot D_{1}$ & Yes & - & - \\
\hline$B_{1} \cdot D_{2}$ & Yes & - & - \\
\hline$B_{2} \cdot D_{1}$ & No & No & - \\
\hline$B_{2} \cdot D_{2}$ & No & No & - \\
\hline$B_{2} \cdot \overline{D_{1,2}}$ & No & Yes & $q_{B_{2}} p_{\mathrm{D}_{1,2}}$ \\
\hline
\end{tabular}

$$
\frac{\partial Q_{2}}{\partial q_{A_{2}}}=q_{B_{2}}-q_{B_{2}} q_{D_{1,2}}=q_{B_{2}}\left(1-q_{D_{1,2}}\right)=q_{B_{2}} p_{D_{1,2}}
$$

This agrees with the expression derived from Table 1.

All derivatives that give the criticality for each component $i$ in each phase $j$ are given in Table 2 .

\subsection{Phase transition failure}

The phase transition function is the likelihood of failure on transition to each phase, $Q_{j}^{\mathrm{T}}$. For each component $i$ that contributes to this phase transition failure there will be a criticality function, $G_{i, j, k}^{\mathrm{T}}$, expressing the probability that the system is in a critical condition such that the failure of component

Table 1 Criticality of component $A$ in phase 2 
Table 2 Birnbaum's measure of importance for each component in each phase

\begin{tabular}{lllll}
\hline & $A$ & $B$ & $C$ & $D$ \\
\hline Phase 1 & $p_{B_{1}}$ & $p_{A_{1}}$ & 0 & 0 \\
Phase 2 & $q_{B_{2}} p_{D_{1,2}}$ & $q_{A_{2}} p_{D_{1,2}}$ & 0 & $p_{A_{1}} p_{B_{1}}-q_{A_{2}} q_{B_{2}}$ \\
Phase 3 & 0 & $p_{A_{1}} q_{C_{1,3}} p_{D_{1,2}}$ & $p_{A_{1,2}} q_{B_{2}} p_{D_{1,2}}+p_{A_{1}} q_{B_{3}} p_{D_{1,2}}$ & $p_{A_{1,2}} p_{B_{1}} p_{C_{1,3}}+p_{A_{1}} p_{B_{1,3}}$ \\
Phase 4 & 0 & 0 & 0 & $+p_{A_{1}} p_{B_{1,2}} p_{C_{1,3}}-p_{A_{1,2}} p_{B_{1,2}} p_{C_{1,3}}$ \\
& & & & $-p_{A_{1}} p_{B_{1,3}} p_{C_{1,3}}$ \\
\hline
\end{tabular}

Table 3 Transition criticality function for the phase $j$ failure for component $i$ in phase $k$

\begin{tabular}{|c|c|c|c|c|c|}
\hline \multirow[b]{2}{*}{$j$} & \multirow[b]{2}{*}{ Phase of component failure $k$} & \multicolumn{4}{|c|}{$i$} \\
\hline & & $A$ & $B$ & $C$ & $D$ \\
\hline Phase 2 & 1 & 0 & 0 & 0 & $p_{A_{1}} p_{B_{1}}$ \\
\hline \multirow[t]{2}{*}{ Phase 3} & 1 & 0 & 0 & $p_{A_{1,2}} q_{B_{2}} q_{D_{1,2}}$ & 0 \\
\hline & 2 & 0 & $p_{A_{1,2}} q_{C_{1,2}} p_{D_{1,2}}$ & $p_{A_{1,2}} q_{B_{2}} p_{D_{1,2}}$ & 0 \\
\hline \multirow[t]{2}{*}{ Phase 4} & 1 & 0 & 0 & 0 & 0 \\
\hline & 2 & 0 & 0 & 0 & 0 \\
\hline
\end{tabular}

$i$ in phase $k$ prior to phase $j$ will cause phase transition failure. This is given by

$$
G_{i, j, k}^{\mathrm{T}}=\frac{\partial Q_{j}^{\mathrm{T}}}{\partial q_{i_{k}}}
$$

The transition criticality function (equation (25)) for each component in the simple phased mission example presented in Fig. 2 for each of the four phases is given in Table 3.

\section{PHASE IMPORTANCE MEASURES}

\subsection{In-phase importance}

Equation (20) gives the criticality measure of importance for component $i$ in a non-phased mission. Extending this to give the importance contribution to the failure of component $i$ in phase $j$ yields

$$
I_{i, j}^{\mathrm{P}}=\frac{G_{i, j} q_{i_{j}}}{Q_{j}}
$$

This in-phase importance measure provides the contribution that the failure of component $i$ makes to causing a failure of the mission during phase $j$. The phase failure can also occur owing to a failure on transition to the phase. Both in-phase and transition failures will contribute to $Q_{j}$. The two importance contributions can, however, be considered individually.

\subsection{Phase transition failure}

Phase transition failure requires that the failure conditions for phase $j$ have occurred in some phase $k$ prior to phase $j$ and that these conditions do not result in any previous phase failure. The transition importance measure, $I_{i, j}^{\mathrm{T}}$, is the failure contribution that component $i$ makes to the transition failure in phase $j$ as a proportion of the total phase failure. The contribution of component $i$ is summed over all the preceding phases to phase $j$, i.e.

$$
I_{i, j}^{\mathrm{T}}=\frac{\sum_{k=1}^{j-1} G_{i, j, k}^{\mathrm{T}} q_{i_{k}}}{Q_{j}}=\frac{\sum_{k=1}^{j-1}\left(\partial Q_{j}^{\mathrm{T}} / \partial q_{i_{k}}\right) q_{i_{k}}}{Q_{j}}
$$

The total importance contribution of component $i$ to the phase $j$ failure is

$$
I_{i, j}=I_{i, j}^{\mathrm{P}}+I_{i, j}^{\mathrm{T}}
$$

\section{MISSION IMPORTANCE MEASURES}

When a system does not reach the required level of performance over a mission, decisions need to be made as to how the system can be improved in order to achieve a better performance. Weak aspects of the system design need to be identified and rectified. Importance measures can aid in the process of identifying the most significant contributions to the system failure. For a phased mission system it is necessary to identify the contribution to failure made by the components with regard to the entire mission, not just any signal phase. The criticality measure over the mission for each component $i$ is given by the proportion of mission failures to which component $i$ contributes

$$
\frac{I_{i}^{\mathrm{M}}=\sum_{j=1}^{n}\left\{\left(\partial Q_{j} / \partial q_{i_{j}}\right) q_{i_{j}}+\left(\sum_{k=1}^{j-1}\left(\partial Q_{j}^{\mathrm{T}} / \partial q_{i_{k}}\right) q_{i_{k}}\right)\right\}}{Q_{\text {miss }}}
$$

where $n$ is the number of phases. 


\section{MISSION PROGRESSION}

For some systems it is possible to track the progress of the mission, for example a satellite mission. As the mission successfully completes its sequence of phases, the mission or phase failure likelihoods can be updated, conditional on the successful completion of each phase.

If the mission has progressed successfully to the end of phase $k$, then the probability of failure, $Q_{j}$ during phases $1 \leqslant j \leqslant k$ is known to be zero. For predictions of phase $j$ failure probability (conditional on having successfully completed phases $1, \ldots, k$ ), $Q_{j \mid \bar{k}}$, we have

$$
\begin{aligned}
\mathrm{Q}_{j \mid \bar{k}}= & P(\text { system failure in phase } j, \\
& \quad \text { given successful completion of phase } k) \\
= & P\left(P h_{j} \mid \overline{P h_{1}} \ldots \overline{P h_{k}}\right) \\
= & \frac{P\left(P h_{j} \cap \overline{P h_{1}} \cap \ldots \cap \overline{P h_{k}}\right)}{P\left(\overline{P h_{1}} \cap \ldots \cap \overline{P h_{k}}\right)} \\
= & \frac{P\left(P h_{j}\right)}{P\left(\overline{P h_{1}} \cap \ldots \cap \overline{P h_{k}}\right)}=\frac{Q_{j}}{1-\sum_{i=1}^{k} Q_{i}}
\end{aligned}
$$

In the same way, the probability of failure on transition to phase $j$ and the probability of in-phase failure in phase $j$, conditional on the successful completion of the first $k$ phases, are

$$
Q_{j \mid \bar{k}}^{\mathrm{T}}=\frac{Q_{j}^{\mathrm{T}}}{1-\sum_{i=1}^{k} Q_{i}}, \quad Q_{j \mid \bar{k}}^{\mathrm{P}}=\frac{Q_{j}^{\mathrm{P}}}{1-\sum_{i=1}^{k} Q_{i}}
$$

The updated mission failure probability is then

$$
Q_{\text {miss }}=\sum_{j=k+1}^{n} Q_{j \mid \bar{k}}
$$

Table 4 Component phase failure probabilities

\begin{tabular}{lllll}
\hline & $A$ & $B$ & $C$ & $D$ \\
\hline Phase 1 & 0.1 & 0.2 & 0.025 & 0.1 \\
Phase 2 & 0.05 & 0.1 & 0.05 & 0.05 \\
Phase 3 & 0.2 & 0.05 & 0.025 & 0.1 \\
Phase 4 & 0.025 & 0.1 & 0.05 & 0.2 \\
\hline
\end{tabular}

\section{SYSTEM EXAMPLE}

Considering the example phased mission system illustrated in Fig. 2 and the component phase failure probabilities given in Table 4, the phase failure probabilities are $Q_{1}=0.28, Q_{2}=0.11275, Q_{3}=0.01105$, and $Q_{4}=0.2106$. These are made up of in-phase and phase transition contributions: $Q_{1}^{\mathrm{P}}=0.28, Q_{2}^{\mathrm{P}}=$ $0.04075, \quad Q_{2}^{\mathrm{T}}=0.072, \quad Q_{3}^{\mathrm{P}}=5.631 \times 10^{-3}, \quad Q_{3}^{\mathrm{T}}=5.419 \times$ $10^{-3}, Q_{4}^{\mathrm{P}}=0.1404$, and $Q_{4}^{\mathrm{T}}=0.0702$. This gives an overall mission failure probability of $Q_{\text {miss }}=0.6144$.

Using equations (26), (27), and (29), the component importance measures throughout the phased mission are given in Table 5.

As the mission successfully completes phases, equations (30) and (32) provide the phase and overall mission failure probabilities which are given in Table 6 .

\section{BINARY DECISION DIAGRAMS - MISSION QUANTIFICATION}

Using the fault tree analysis approach reported above provides a systematic way of analysing the phased mission and obtaining the component importance contributions. However, as the complexity of the mission fault trees increases and the number of phases increases, the calculations become difficult to perform in a realistic time. There are two reasons for this: first, the computational effort to determine the phase failure modes (prime implicants) can be high for large problems; second, then using the prime implicants to calculate the phase failure probability from equation (4) can also be computationally intensive. Since the fault trees are non-coherent, many terms in the series expansion may need to be evaluated to obtain convergence, and approximations

\begin{tabular}{|c|c|c|c|c|c|c|c|c|}
\hline Component & $\begin{array}{l}\text { In-phase } 1 \\
\text { import }\end{array}$ & $\begin{array}{l}\text { Transition to } \\
\text { phase } 2 \text { import }\end{array}$ & $\begin{array}{l}\text { In-phase } 2 \\
\text { import }\end{array}$ & $\begin{array}{l}\text { Transition to } \\
\text { phase } 3 \text { import }\end{array}$ & $\begin{array}{l}\text { In-phase } 3 \\
\text { import }\end{array}$ & $\begin{array}{l}\text { Transition to } \\
\text { phase } 4 \text { import }\end{array}$ & $\begin{array}{l}\text { In-phase } 4 \\
\text { import }\end{array}$ & $\begin{array}{l}\text { Total mission } \\
\text { import }\end{array}$ \\
\hline$A$ & 0.2857 & 0 & 0.0377 & 0 & 0 & 0 & 0 & 0.1371 \\
\hline$B$ & 0.6429 & 0 & 0.0377 & 0.4904 & 0.3462 & 0 & 0 & 0.3149 \\
\hline$C$ & 0 & 0 & 0 & 0.04904 & 0.25 & 0 & 0 & 0.1799 \\
\hline$D$ & 0 & 0.6386 & 0.3171 & 0 & 0 & 0.3333 & 0.6667 & 0.5181 \\
\hline
\end{tabular}
used for coherent fault trees may not be appropriate. A practical method needs to be established in order to enable the analysis of real systems.

Table 6 Phase progression failure probabilities

\begin{tabular}{llllll}
\hline & $Q_{1}$ & $Q_{2}$ & $Q_{3}$ & $Q_{4}$ & $Q_{\text {miss }}$ \\
\hline 0 & 0.28 & 0.11275 & 0.01105 & 0.2106 & 0.6144 \\
1 & - & 0.1566 & 0.01535 & 0.2925 & 0.4645 \\
2 & - & - & 0.01820 & 0.3468 & 0.3650 \\
3 & - & - & - & 0.3532 & 0.3532
\end{tabular}

Table 5 Mission importance contributions 
For conventional systems analysis, the use of binary decision diagrams (BDDs) [11-13] offers a fast, efficient, and accurate means of analysing fault trees, coherent or non-coherent. BDDs were used in the context of a phased mission analysis in reference [3].

The fault trees for each phase failure of the example mission are given in Fig. 2. The causes of failure in each phase accounting for the survival of previous phases are obtained using the fault tree shown in Fig. 1. Converting these formulations to BDDs gives the BDDs for the failure of phases 2 to 4 in Figs 3(a) to (c) respectively (the phase 1 failure fault tree and BDD are trivial). They have been constructed assuming all component failures in each of the phases are independent. The construction process has been performed using a component ordering of $A_{1}<A_{2}<A_{3}$ $<A_{4}<B_{1}<B_{2}<\ldots<D_{3}<D_{4}$ and the conversion rules given in reference $[\mathbf{1 1}]$ which produce the BDDs in an ite (if-then-else) structure.

By convention, all left branches leaving each node are 1-branches, and all right branches are
0 -branches. The BDDs give the causes of each phase failure in a disjoint (mutually exclusive) form for causes of both in-phase and phase transition failure. To calculate the in-phase failure probability, the causes of phase transition failure are removed from the causes of failure obtained from the BDD, and the probability of the remaining causes (paths) summed. To obtain the phase failure causes from the BDD, each path to a terminal-1 node is considered and all component conditions (working and failed) are listed. Considering the BDD for phase 2 (Fig. 3(a)) gives

$$
\begin{aligned}
& A_{2} B_{2} \\
& A_{2} \bar{B}_{1,2} D_{1,2} \\
& \bar{A}_{1,2} \bar{B}_{1} D_{1,2}
\end{aligned}
$$

Expanding the second and third causes in the list to separate those causes with $D$ failing in phase 1 (and causing phase transition failure) and those
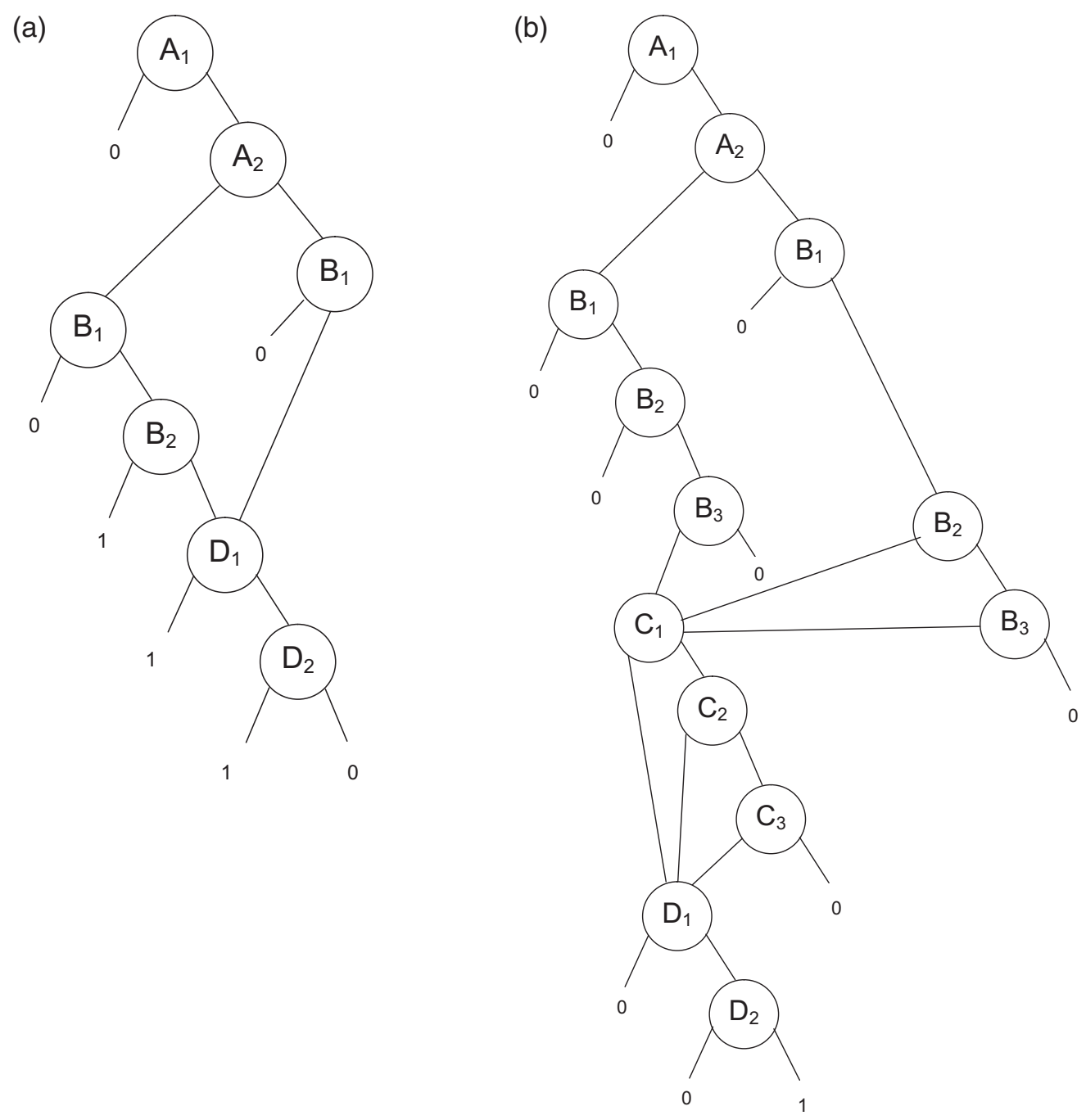

Fig. 3 (a) BDD for phase 2 failure; (b) BDD for phase 3 failure; ((c) overleaf) 


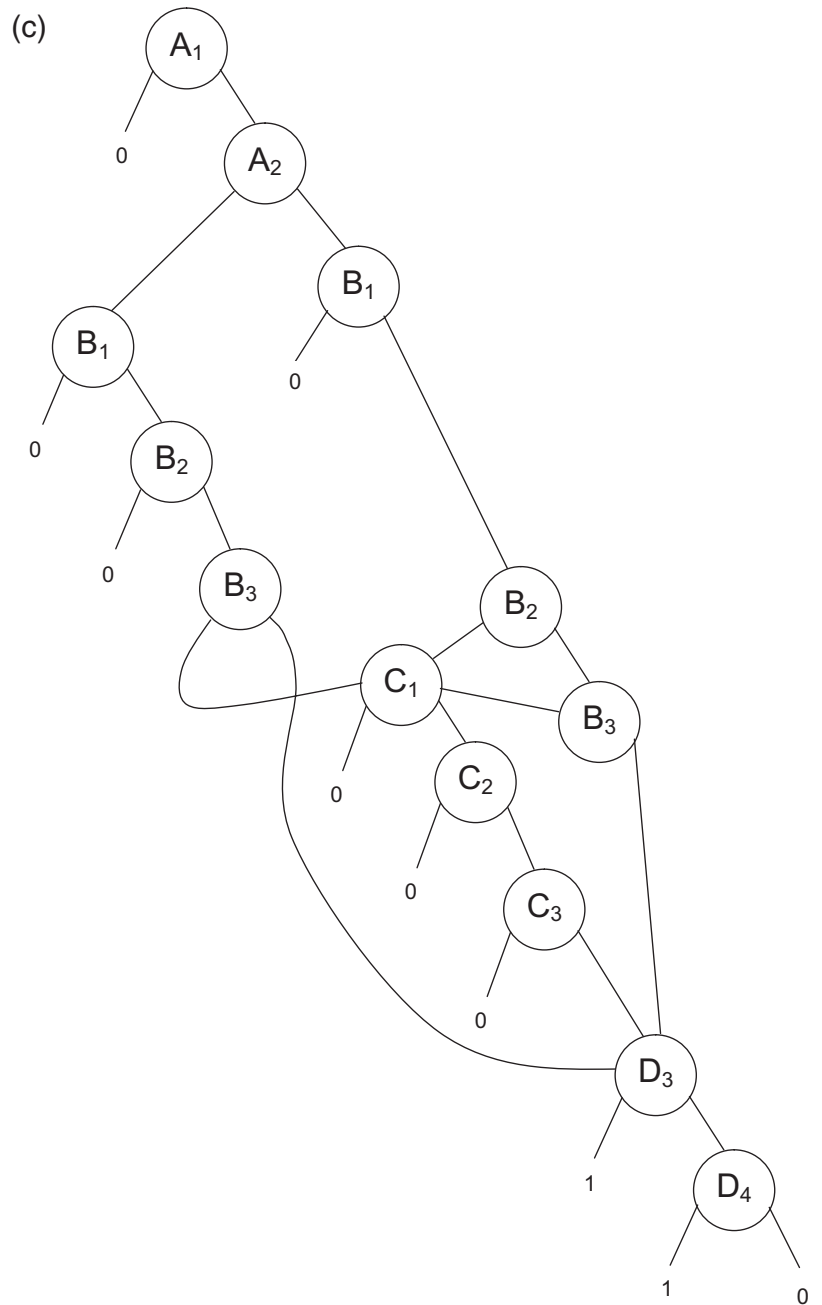

Fig. 3 (c) BDD for phase 4 failure

with $D$ failing in phase 2 (and causing in-phase failure) gives

$$
\begin{aligned}
& A_{2} B_{2} \\
& A_{2} \bar{B}_{1,2} D_{1} \times(\text { transition failure }) \\
& A_{2} \bar{B}_{1,2} D_{2} \\
& \bar{A}_{1,2} \bar{B}_{1} D_{1} \times(\text { transition failure }) \\
& \bar{A}_{1,2} \bar{B}_{1} D_{2}
\end{aligned}
$$

The second and fourth terms are removed, as they cause phase transition failure. Those that are left not only cause an in-phase failure but also are in a disjoint form, so $Q_{2}^{\mathrm{P}}$ is obtained by summing the path probabilities, giving 0.04075 .

The phase transition fault trees, whose causes are given in equations (11) to (13) are converted to the BDDs shown in Figs 4 (a) to (c) for phases 2 to 4 respectively using the same variable ordering.

From Fig. 4(a) the single cause of failure on transition to phase 2 is $\bar{A}_{1} \bar{B}_{1} D_{1}$, the probability of which is 0.072 , which gives $Q_{2}^{\mathrm{T}}$. The likelihood of failure in phase 2 is therefore 0.11275 , as obtained using the previous fault tree method by summing $Q_{2}^{\mathrm{P}}+Q_{2}^{\mathrm{T}}$.

For phases 3 and 4, the phase failure BDDs given in Figs 3(b) and (c) produce the failure causes shown below:

Phase 3 failure

$$
\begin{aligned}
& A_{2} B_{3} C_{1,3} \bar{D}_{1,2} \quad A_{2} B_{3} C_{1,3} \bar{D}_{1,2} \\
& \bar{A}_{1,2} B_{2,3} C_{1,3} \bar{D}_{1,2} \Rightarrow \bar{A}_{1,2} B_{2} C_{1,2} \bar{D}_{1,2} \\
& \times \text { (transition failure) } \\
& \bar{A}_{1,2} B_{2} C_{3} \bar{D}_{1,2} \\
& \bar{A}_{1,2} B_{3} C_{1,3} \bar{D}_{1,2}
\end{aligned}
$$

Phase 4 failure

$$
\begin{aligned}
A_{2} B_{3} \bar{C}_{1,3} D_{3,4} \quad & A_{2} B_{3} \bar{C}_{1,3} D_{3} \\
& \times(\text { transition failure }) \\
A_{2} \bar{B}_{1,3} D_{3,4} \Rightarrow & A_{2} B_{3} \bar{C}_{1,3} D_{4} \\
\bar{A}_{1,2} B_{2,3} \bar{C}_{1,3} D_{3,4} & A_{2} \bar{B}_{1,3} D_{3} \\
& \times(\text { transition failure }) \\
\bar{A}_{1,2} \bar{B}_{1,3} D_{3,4} \quad & A_{2} \bar{B}_{1,3} D_{4} \\
& \bar{A}_{1,2} B_{2,3} \bar{C}_{1,3} D_{3} \times(\text { transition failure }) \\
& \bar{A}_{1,2} B_{2,3} \bar{C}_{1,3} D_{4} \\
& \bar{A}_{1,2} \bar{B}_{1,3} D_{3} \times(\text { transition failure }) \\
& \bar{A}_{1,2} \bar{B}_{1,3} D_{4}
\end{aligned}
$$

Summing the probabilities of the disjoint paths left after the transition failure causes have been removed gives $Q_{3}^{\mathrm{P}}$ and $Q_{4}^{\mathrm{P}}$.

The phase transition failure BDDs given in Figs 4 (b) and (c) produce the causes shown below.

Phase 3 transition failure

$$
\bar{A}_{1,2} B_{2} C_{1,2} \bar{D}_{1,2}
$$

Phase 4 transition failure

$$
\begin{aligned}
& A_{2} B_{3} \bar{C}_{1,3} D_{3} \\
& A_{2} \bar{B}_{1,3} D_{3} \\
& \bar{A}_{1,2} B_{2,3} \bar{C}_{1,3} D_{3} \\
& \bar{A}_{1,2} \bar{B}_{1,3} D_{3}
\end{aligned}
$$

Summing the likelihood of these paths gives $Q_{3}^{\mathrm{T}}$ and $Q_{4}^{\mathrm{T}}$.

\section{TERNARY DECISION DIAGRAMS - BIRNBAUM'S IMPORTANCE QUANTIFICATION}

While the BDDs can be used to calculate the phase failure probabilities, they do not enable the calculation of the component importance measures. All of the component importance measures developed in this paper are based on Birnbaum's measure of importance, either in-phase, $\partial Q_{j} / \partial q_{i_{j}}$, or phase transition, $\partial Q_{j}^{\mathrm{T}} / \partial q_{i_{k}}$. For these derivatives to yield the correct importance measures, $Q_{j}$ and $Q_{j}^{\mathrm{T}}$ need to be expressed in Henley and Inagaki's formulation. 
(a)

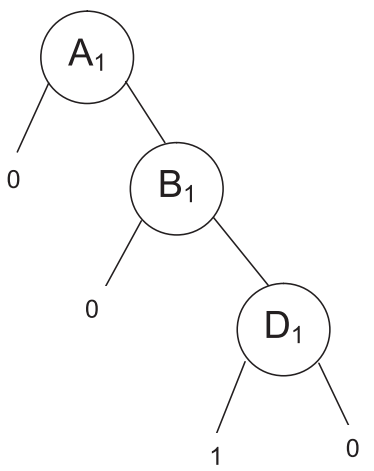

(b)

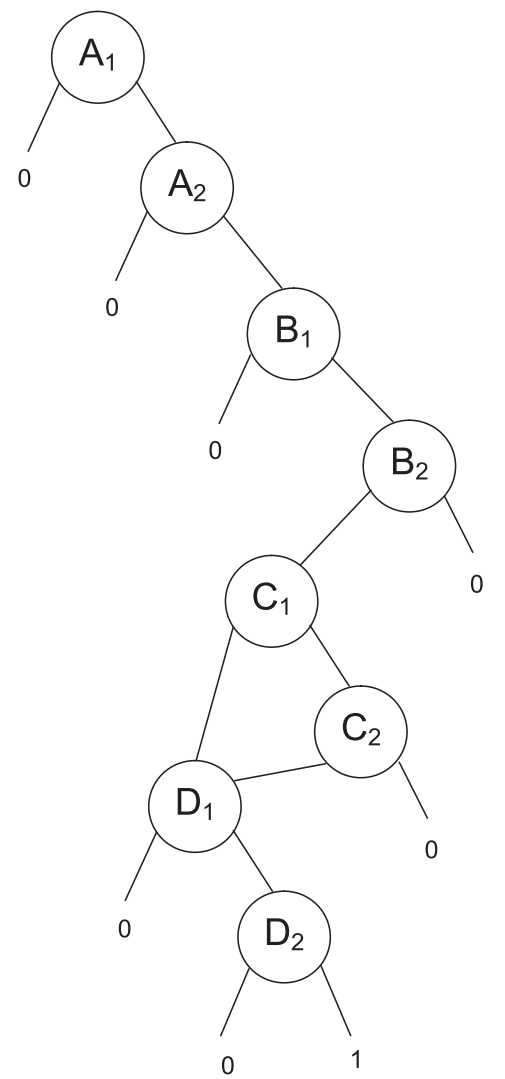

(c)

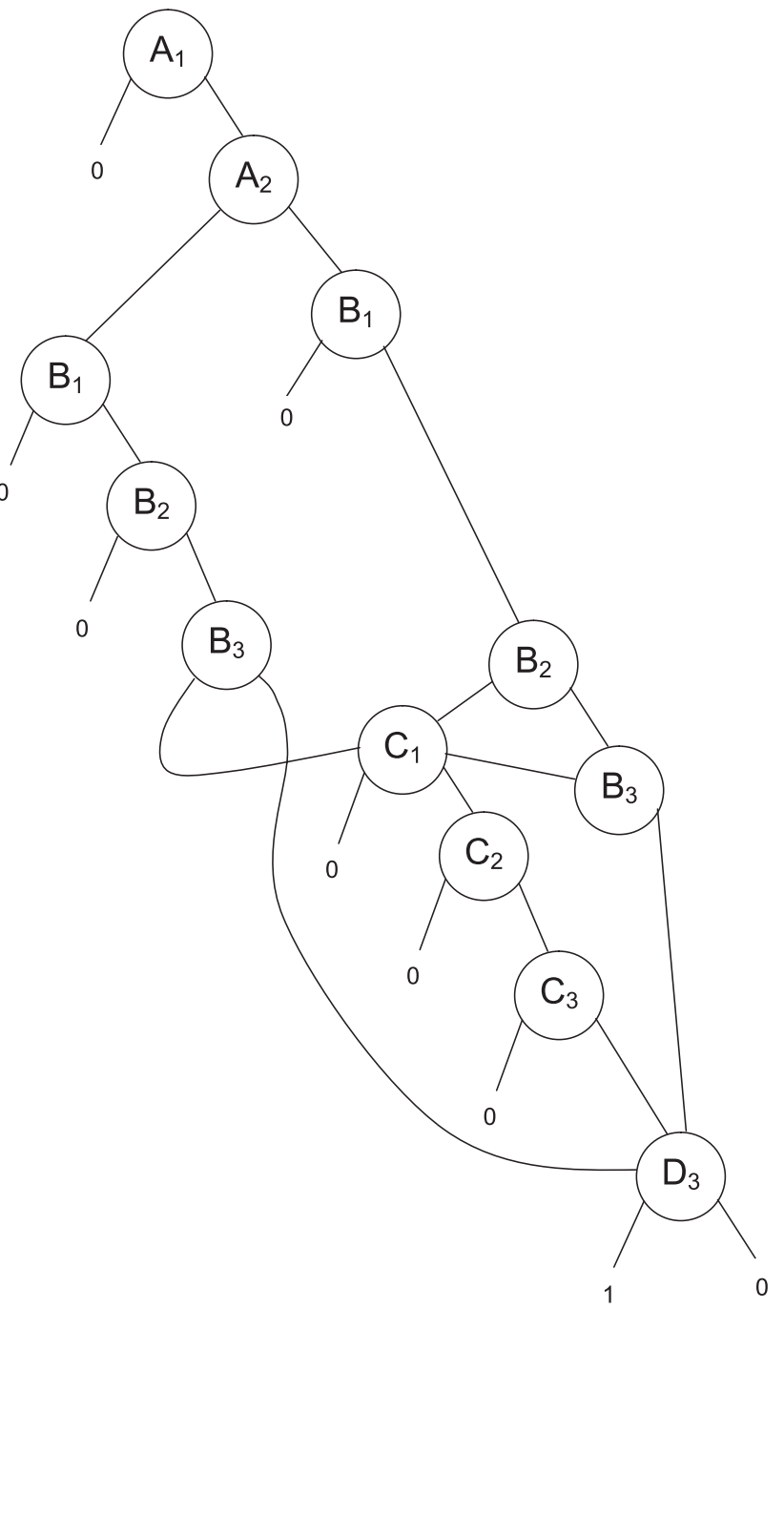

Fig. 4 (a) BDD for phase 2 transition failure; (b) BDD for phase 3 transition failure; (c) BDD for phase 4 transition failure

The negated success terms in the phase failure modes occur purely to ensure the conditions for the successful completion of the previous phases. As success events enable progression to the phase, they do not cause phase failure. As such, for variable $x_{i}$ they are assigned a variable $p_{x_{i}}$ and failure events $q_{x i}$, which are assumed to be independent in order for the derivatives to give the correct contribution. When the BDDs are constructed, the process effectively converts all $p_{x_{i}}$ terms to $1-q_{x_{i}}$, and so the derivatives no longer deliver Birnbaum's measure of importance.
Using the Henley and Inagaki formulation, $Q_{j}$ can be expressed as

$$
Q_{j}=q_{i_{j}} Q_{A}+p_{i_{j}} Q_{B}+Q_{C}
$$

The three terms represent those products involving the failure of component $i$ in phase $j$, the functioning of component $i$ in phase $j$, and those terms for which $i$ is irrelevant in phase $j$

$$
G_{i_{j}}=\frac{\partial Q_{j}}{\partial q_{i_{j}}}=Q_{A}
$$

which is the Birnbaum importance measure that needs to be calculated. 
From equation (39) the BDD probability function below the 1 -branch of node $i_{j}$ is

$$
Q_{j}\left(q_{i_{j}}=1\right)=Q_{A}+Q_{C}
$$

Thus, it is necessary to be able to calculate $Q_{C}$ to subtract from this to obtain $G_{i_{j}}$ (equation (40)). Considering now the logic form of the BDD, there is (for first variable $i_{j}$ )

$$
P h_{j}=i_{j} P h_{A}+\bar{i}_{j} P h_{B}
$$

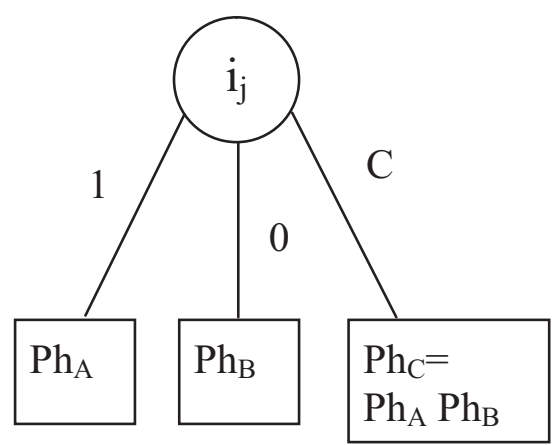

Fig. 5 Ternary decision diagram

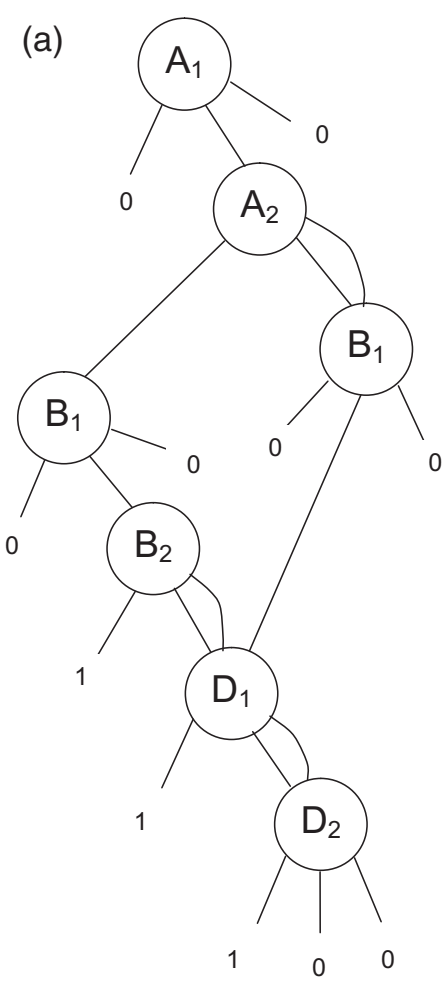

By consensus

$$
P h_{j}=i_{j} P h_{A}+\overline{i_{j}} P h_{B}+P h_{A} P h_{B}
$$

so that

$$
Q_{C}=P\left[P h_{A} P h_{B}\right]
$$

For the quantification of Birnbaum's measure of 'failure importance' for non-coherent systems [15], the system structure needs to be expressed as a ternary decision diagram (TDD).

The TDD structure shown in Fig. 5 is where the consensus branch is obtained from equation (43). In this form of diagram, a third 'consensus' branch from each BDD node is introduced which develops the causes of failure for which the variable $i_{j}$ is irrelevant. It is constructed by forming the conjunction of the logic functions on the 1-branch AND 0-branch of the nodes. Adding the consensus branch to the BDDs in Figs 3(a) to (c) results in the TDDs in Figs 6(a) to (c). The convention used for all the TDDs is that the left node is the 1-branch, the centre node is the 0 -branch, and the right branch is the consensus C-branch.

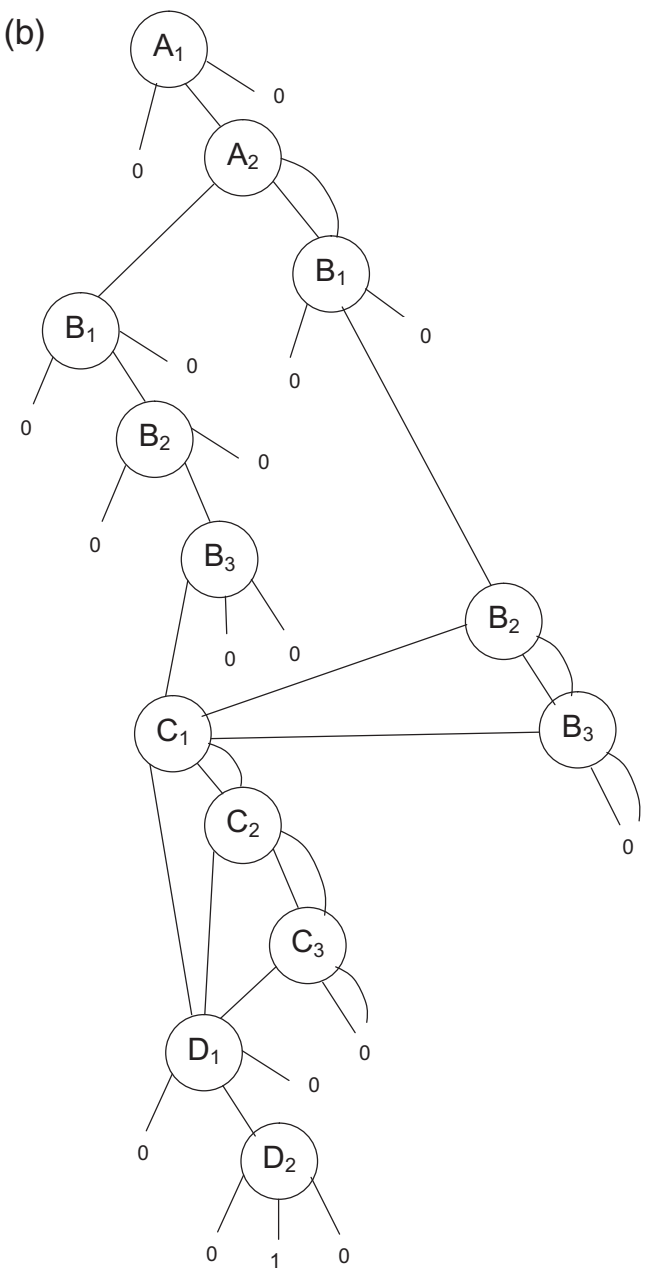

Fig. 6 (a) TDD for phase 2 failure; (b) TDD for phase 3 failure 


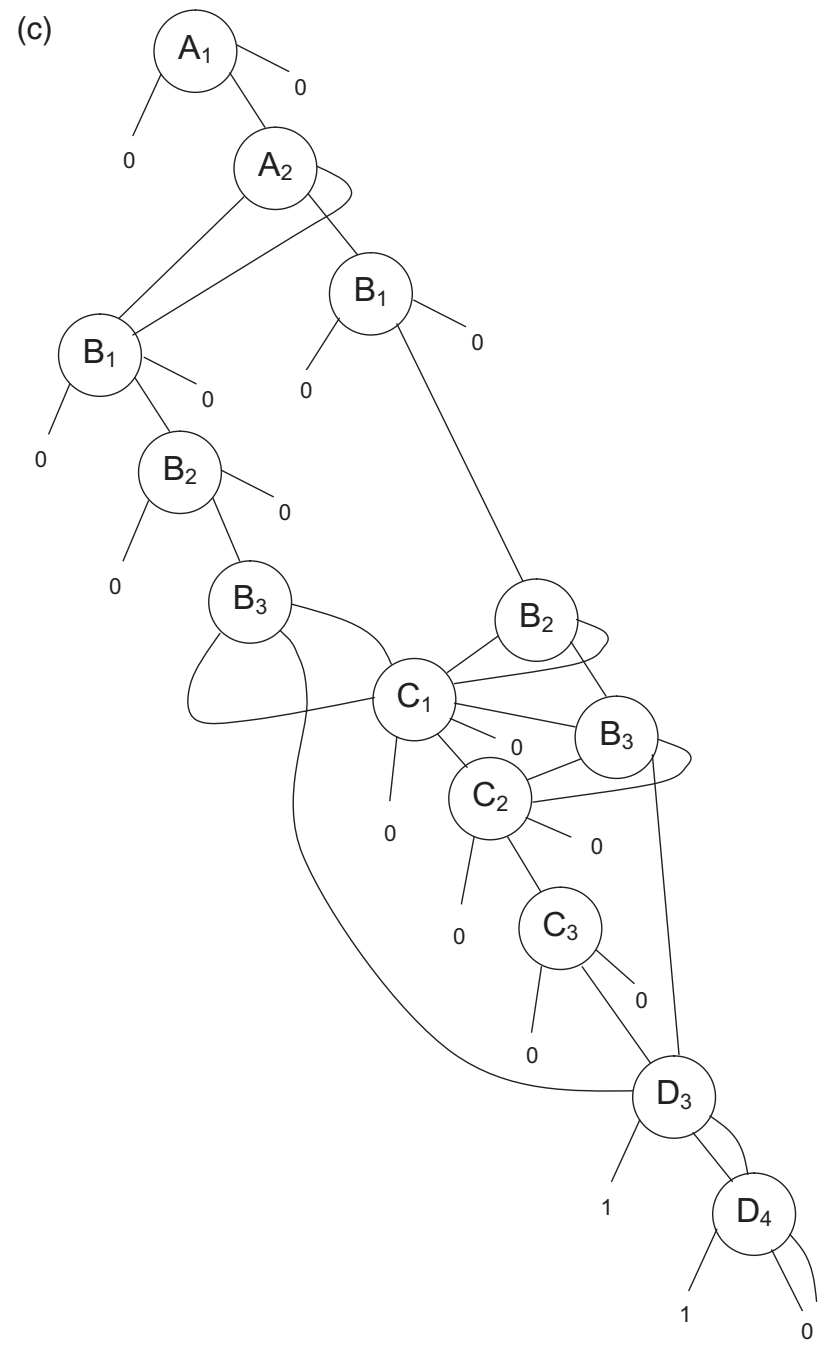

Fig. 6 (c) TDD for phase 4 failure

\subsection{Birnbaum's phase criticality measure}

Birnbaum's phase criticality measure for component $i$ in phase $j$ is calculated from the TDD using

$$
\begin{aligned}
G_{i, j} & =P\left(P h_{A}\right)-P\left(P h_{B}\right) \\
& =Q_{j}\left(i_{j}=1, \boldsymbol{q}\right)-Q_{j}\left(i_{j}={ }^{\prime}-', \boldsymbol{q}\right)
\end{aligned}
$$

Considering the structure of the TDD

$$
\begin{aligned}
& Q_{j}\left(i_{j}=1, \boldsymbol{q}\right)= \sum_{\substack{\text { all } \\
\text { nodes } \\
i_{j}}}\left(p r_{i_{j}}(\boldsymbol{q}) \cdot p o_{i_{j}}^{1}(\boldsymbol{q})\right)+Z(\boldsymbol{q}) \\
& Q_{j}\left(i_{j}={ }^{\prime}-', \boldsymbol{q}\right)=\sum_{\substack{\text { all } \\
\text { nodes } \\
i_{j}}}\left(p r_{i_{j}}(\boldsymbol{q}) \cdot p o_{i_{j}}^{\mathrm{C}}(\boldsymbol{q})\right)+Z(\boldsymbol{q})
\end{aligned}
$$

giving

$$
\begin{aligned}
G_{i, j}= & \sum_{\substack{\text { all } \\
\text { nodes }\\
}} p r_{i_{j}}(q)\left[p o_{i_{j}}^{1}(\boldsymbol{q})-p o_{i_{j}}^{\mathrm{C}}(\boldsymbol{q})\right]
\end{aligned}
$$

where

$\operatorname{pr}_{i_{j}}(\boldsymbol{q})=$ probability of the path section from the root node to node $i_{j}$

$p o_{i_{j}}^{1}(\boldsymbol{q})=$ probability of the path section from the 1branch of node $i_{j}$ to a terminal-1 node

$p o_{i_{j}}^{\mathrm{C}}(q)=$ probability of the path section from the consensus branch of node $i_{j}$ to a terminal-1 node $Z_{(q)}=$ probability of paths from the root node to a terminal-1 node not passing through a node of variable $i_{j}$

As an example, consider the calculation of $G_{C, 3}$ using the TDD in Fig. 6(b).

The failure of component $C$ in phase 3 , causing phase 3 failure, is of interest. Therefore, $C$ cannot fail in phases 1 or 2, so $C_{1}=C_{2}=0$ in the TDD.

The component conditions from the root node to node $C_{3}$ are

$$
A_{2} B_{3}+\bar{A}_{1,2} B_{2,3}
$$

giving

$$
\begin{aligned}
& p r_{C_{3}}=q_{A_{2}} q_{B_{3}}+p_{A_{1,2}} q_{B_{2,3}} \\
& p o_{C_{3}}^{1}=p_{D_{1,2}} \\
& p o_{C_{3}}^{\mathrm{C}}=0
\end{aligned}
$$

so that

$$
\begin{aligned}
\frac{\partial Q_{3}}{\partial q_{C_{3}}} & =\left(q_{A_{2}} q_{B_{3}}+p_{A_{1,2}} q_{B_{2,3}}\right) p_{D_{1,2}} \\
& =\left(p_{A_{1,2}} q_{B_{2}}+p_{A_{1,2}} q_{B_{3}}+q_{A_{2}} q_{B_{3}}\right) p_{D_{1,2}} \\
& =\left(p_{A_{1,2}} q_{B_{2}}+p_{A_{1}} q_{B_{3}}\right) p_{D_{1,2}}
\end{aligned}
$$

which agrees with the entry in Table 2 .

The TDDs are quantified by the above process, which only considers the 1-branches and 0 -branches leaving any node. The consensus C-branch is only considered to evaluate $p o_{i_{j}}^{\mathrm{C}}(\boldsymbol{q})$.

\subsection{Birnbaum's phase transition criticality measure}

Birnbaum's measure of phase transition criticality, $G_{i, j, k}^{\mathrm{T}}$, due to the failure of component $i$ in phase $k$ causing failure in phase $j$ was derived from equation (25). Adding the consensus branch to the phase transition BDDs shown in Figs 4(a) to (c) gives the TDDs shown in Figs 7(a) to (c) for phases 2 to 4 respectively. 
(a)

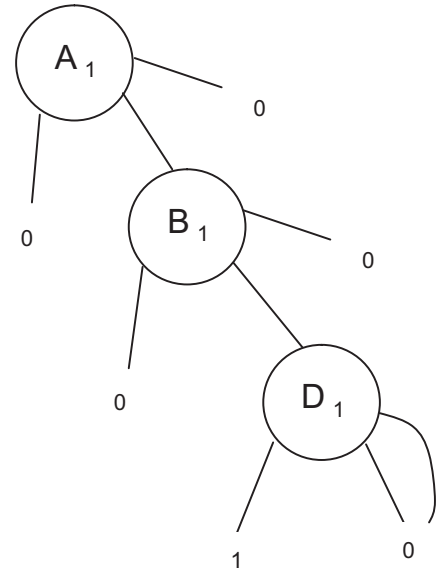

(b)

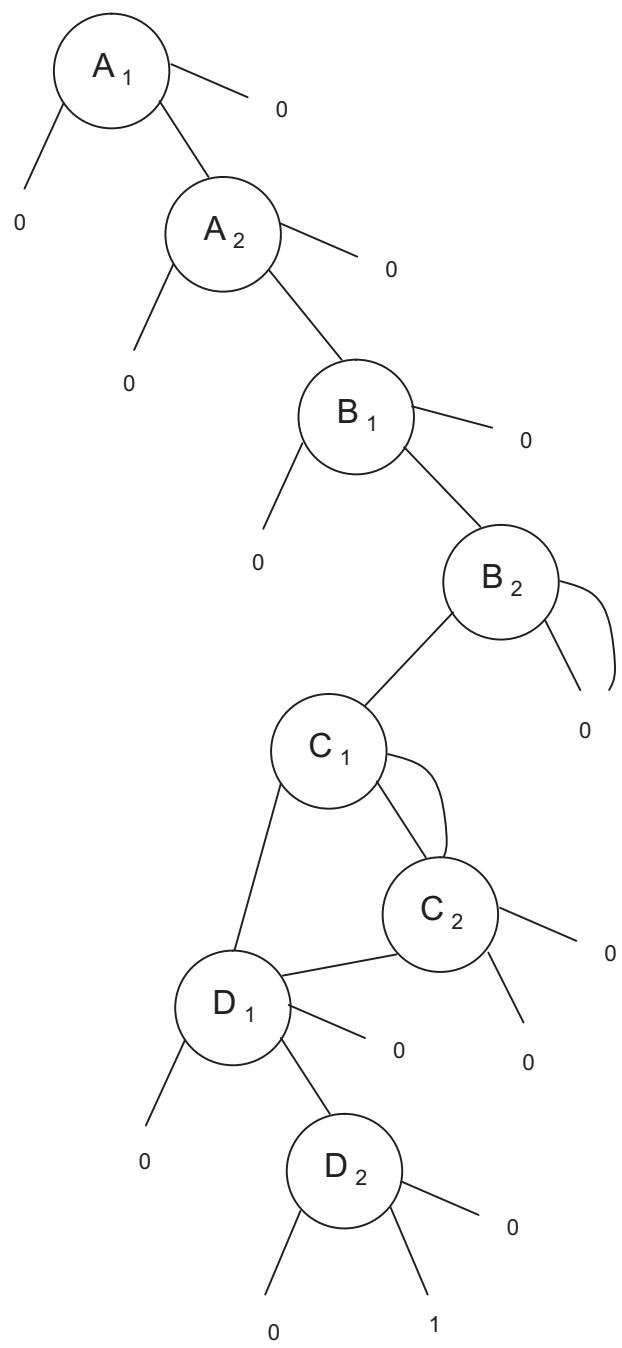

(c)

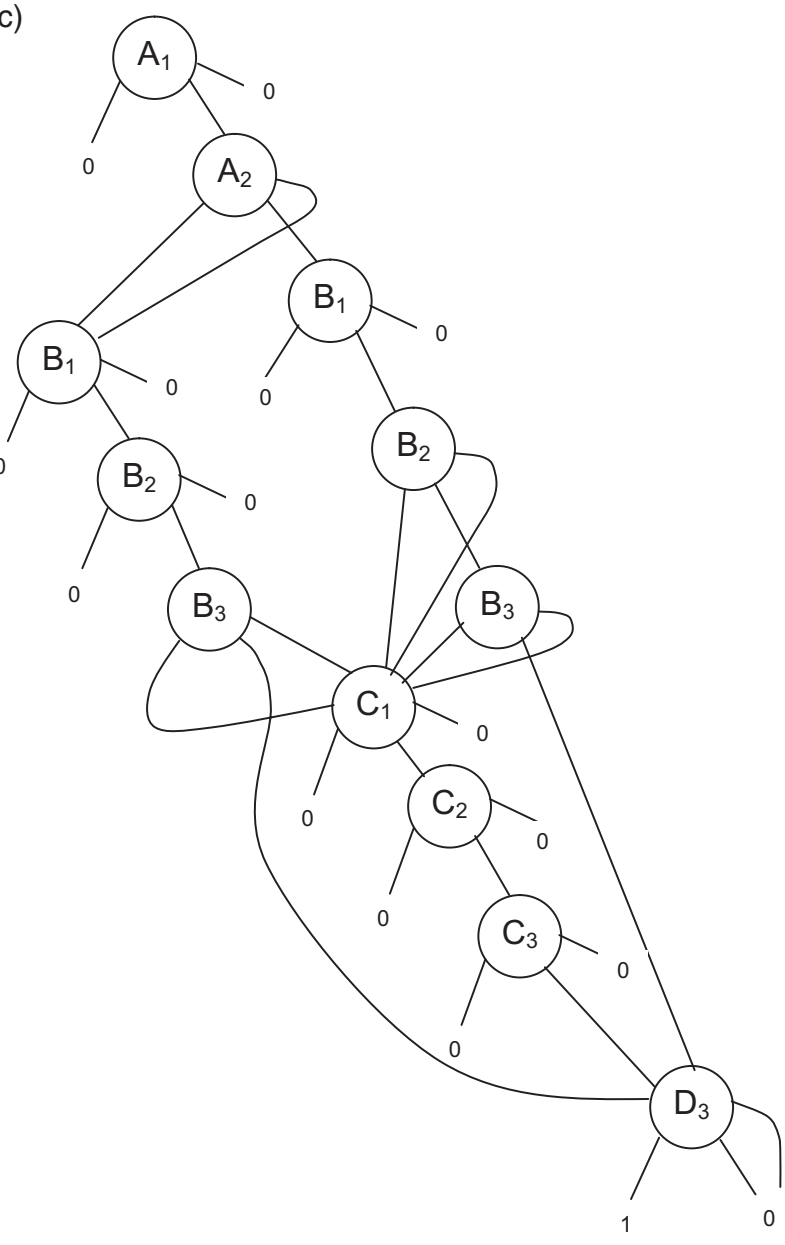


From the 1-branch from $B_{2}$ to a terminal requires $C_{1,2} \bar{D}_{1,2}$

$$
\begin{aligned}
& p o_{B_{2}}^{1}=q_{C_{1,2}} p_{D_{1,2}} \\
& p o_{B_{2}}^{\mathrm{C}}=0 \\
& G_{B, 3,2}^{\mathrm{T}}=p_{A_{1,2}} q_{C_{1,2}} p_{D_{1,2}}
\end{aligned}
$$

As the TDDs are just an extension of the BDDs, they can, of course, be used for phase failure quantification in addition to calculating Birnbaum's measure of importance. To calculate the phase failure probability from the TDDs, the consensus branches are totally ignored. The quantities required to perform a phased mission reliability analysis, including Birnbaum and criticality measures of component importance, can all be calculated from the TDDs formed from the fault tree structures representing phase failure and phase transition failure.

\section{CONCLUSIONS}

A phased mission modelling approach has been presented. A means to evaluate the contribution made by each component to phase and mission failure has been developed. The method enables the phase and mission failure likelihood predictions to be updated as phases of the mission are successfully completed.

In order for the quantities required to be calculated in a reasonable time, a ternary decision diagram (TDD) representation and quantification process has been developed.

\section{REFERENCES}

1 Esary, J. D. and Ziehms, H. Reliability of phased missions. (Eds G. Apostolakis, S. Garriba, and G. Volta), In Reliability and fault-tree analysis, 1975, pp. 213-236 (Society for Industrial Applied Mathematics, Philadelphia, Pennsylvania).

2 Burdick, G. R., Fussell, J. B., Rasmuson, D. M., and Wilson, J. R. Phased mission analysis: a review of new developments and an application. IEEE Trans. Reliability, 1977, R-26, April, 43-49.

3 La Band, R. and Andrews, J. D. Phased mission modelling using fault tree analysis. Proc. Instn Mech. Engrs, Part E: J. Process Mechanical Engineering, 2004, 218, 83-91.

4 Clarotti, C. A., Contini, S., and Somma, R. Repairable multiphase systems-Markov and fault-tree approaches for reliability evaluation. In (Eds G. Apostolakis, S. Garriba, and G. Volta). Synthesis and analysis methods for safety and reliability studies, 1980, pp. 45-58 (Plenum Press, New York).

5 Smotherman, M. and Zemoudeh, K. A nonhomogeneous Markov model for phased mission reliability analysis. IEEE Trans. Reliability, 1989, 38, December, 585-590.

6 Smotherman, M. and Geist, R. M. Phased effectiveness using a nonhomogeneous Markov reward model. Reliability Engng and Syst. Safety, 1990, 27, 241-255.

7 Andrews, J. D. Identifying the major contributions to risk in phased missions. Proceedings of the Annual Reliability and Maintainability Symposium, Newport Beach, California, 23-26 January 2006.

8 Andrews, J. D. Measures of component contribution to the failure of phased missions. Proceedings of the European Safety and Reliability Conference 2006 (ESREL 2006), Estoril, 18-22 September, Safety and reliability for managing risk (Eds C. Guedes Soares and E. Zio), 2006 (Taylor \& Francis, London).

9 Birnbaum, Z. W. On the importance of different components in a multi-component system. Multivariate analysis 11 (Ed. P. R. Krishnaiah), 1969 (Academic Press, New York).

10 Andrews, J. D. and Moss, T. R. Reliability and risk assessment, 2002 (Professional Engineering Publishing Ltd, Bury St Edmunds and London).

11 Rauzy, A. New algorithms for fault tree analysis. Reliability Engng and Syst. Safety, 1993, 40, 203-211.

12 Sinnamon, R. M. and Andrews, J. D. Improved efficiency in qualitative fault tree analysis. Quality and Reliability Engng Int., 1997, 13, 293-298.

13 Sinnamon, R. M. and Andrews, J. D. Improved accuracy in quantitative fault tree analysis. Quality and Reliability Engng Int., 1997, 13, 285-292.

14 Henley, E. J. and Inagaki, T. Probabilistic evaluation of prime implicants and top events for non-coherent systems. IEEE Trans. Reliability, 1980, R-29(5), December.

15 Andrews, J. D. and Beeson, S. Birnbaum's measure of importance for non-coherent Systems. IEEE Trans. Reliability, 2003, 52(2), June, 213-220. 